\title{
Korean mistletoe lectin enhances natural killer cell cytotoxicity via upregulation of perforin expression
}

\author{
Younghoon Kim, ${ }^{1}$ Inbo Kim, ${ }^{1,2}$ Choon-Ho Park, ${ }^{2}$ Jong Bae Kim ${ }^{1,2, *}$
}

\begin{abstract}
Background: Natural killer (NK) cells are crucial components of the innate immune system, providing the first line of defense against pathogens. In a previous study, we demonstrated prophylactic activity of water extract of Korean mistletoe (Viscum album coloratum) on tumor metastasis. However, the leading compound from water extract of Korean mistletoe was not clearly addressed.

Objective: The purpose of this research was mainly focused on addressing the effect of Korean mistletoe lectin (KMLC) on NK cell cytotoxicity, and the ability of cytokine secretion as well as its signal transduction, mitogen-activated protein kinase (MAPK) pathway.

Methods: KMLC was used to test NK cell-mediated cytotoxicity in vitro and in vivo. Non-isotope cytotoxicity assay (bis-N,N,N',N'-tetraacetic acid (BATDA) release assay) was performed to test the cytotoxicity of NK cells against target tumor cells. Receptor expression was checked by flow cytometry analysis and MAPK signal molecules were analyzed by immunoblotting.

Results and Conclusions: KMLC at $200 \mathrm{ng} / \mathrm{mL}$ increased the cytotoxicity of NK92 cells by 35\% compared with untreated cells. KMLC-treated (at $100 \mathrm{ng} / \mathrm{mL}$ ) mice splenocytes showed a 20\% increase in cytotoxic activity. Also, the B chain, one of the subchains of KMLC, increases perforin expression. We demonstrated that the signal transduction controlling NK cell cytotoxicity was mediated by upregulation of the NKG2D receptor and expression of a cytotoxic effector molecule. These results suggested that KMLC possessed immunological activity, mediated by NK cell activation.
\end{abstract}

Keywords: Korean mistletoe, lectin, natural killer cell, cytotoxicity, perforin.

Abbreviations: NK cell, natural killer cell; E:T, effectors to target; MAPK, mitogen-activated protein kinase.

From:

a School of Life Science, Handong Global University, Pohang, Gyeongbuk 79170, Republic of Korea

b Mistle Biotech Co., Ltd., Pohang Technopark, Pohang, Gyeongbuk 79083, Republic of Korea

\section{Introduction}

For centuries, European mistletoe (EM; Viscum album) have been used as medicine. ${ }^{1}$ Anti-tumor and immunomodulatory activities of EM extracts have been reported. ${ }^{2}$ EM extracts have many biological effects such as enhancement of natural killer (NK) cell activity, immunoadjuvant activity, and antitum or activity. ${ }^{3,4}$ It was reported that the antitumor activity of EM was tightly related to its enhancement of NK and lymphokine -activated killer cell activity and macrophage activation. ${ }^{5}$ The active compound in EM extracts was determined to be lectin. ${ }^{6}$ This consists of A and B subchains linked by disulfide bonds, and the amino acid sequence of lectin has been completely

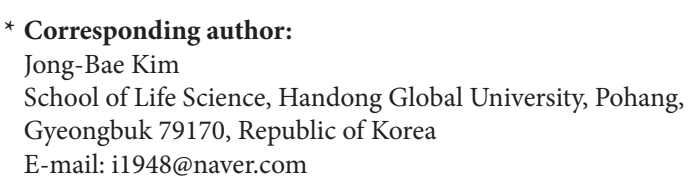

determined. ${ }^{7}$

Korean mistletoe (KM; Viscum album coloratum) is a therapeutic herb that has traditionally been used as a sedative. Recent reports have suggested that KM extracts have immunoadjuvant activity, enhancing cellular and humoral immunity and NK cell activity. ${ }^{8}$ According to a recent study, KM was more cytotoxic than EM against various tumor cells. ${ }^{9}$ Furthermore, KM extract was reported to have a prophylactic effect through its enhancement of NK cell function. ${ }^{8}$ However, the major compound within $\mathrm{KM}$ was not examined, and the underlying cellular mechanisms influencing NK cell activity 
were unclear. ${ }^{8}$ Even though the lectin fraction of KM extract was shown to be one of the active ingredients with tumor -suppressing activity, the underlying cellular mechanisms involved have not been fully studied in vitro.

In order to examine the effects of KM extracts on NK cell cytotoxicity, we isolated KM lectin (KMLC) from water extracts of KM. Lectin is a major component of mistletoe extracts, and KMLC has different structural properties than EM lectin. ${ }^{10}$ KMLC has various biological activities such as cytokine induction, mucosal immunity enhancement, antitumor activity, and immunoadjuvant activity. ${ }^{11-14}$ It is composed of two subunits that are linked by a disulfide bond. ${ }^{15} \mathrm{NK}$ cells are innate immune lymphocytes that are classified as large granular immune cells; these lack surface immunoglobulin and CD3, while expressing CD16 and CD56. ${ }^{16,17}$ NK cells mediate the killing of certain virus-infected and tumor cells, providing a first line of defense against pathogens. ${ }^{18,19}$ Exocytosis of cytolytic granules containing granzyme-B and perforin is essential for NK cell cytotoxicity and represents their primary means of killing target cells. ${ }^{20}$ It is well known that the expression of granzyme- $B$ and perforin is regulated by mitogen activated protein kinase (MAPK) pathway phosphorylation and activation of this pathway in turn is regulated by phosphorylation of protein tyrosine kinase..$^{21,22}$

The present study demonstrated that KMLC had antitumor activity via enhancement of perforin expression and NK cell cytotoxicity. KMLC activated NK92 cells, through effects on MAPK, increased perforin expression and augmented cytotoxic activity against K562 and Yac-1 tumor cell lines. Cytotoxic activity was measured using the bis(acetoxymethyl)-2,2'-6',2" -terpyridine-6,6"-dicarboxylate (BATDA) release assay, which has various advantages over the chromium release assay. ${ }^{23}$

\section{Materials and Methods Cell culture and reagents}

The NK92 tumor cell line was grown in alpha-minimal essential medium (MEM, GIBCO) containing $100 \mathrm{U} / \mathrm{mL}$ recombinant human interleukin-2 (IL-2; PeproTech), 12.5\% fetal bovine serum (GIBCO), and 12.5\% horse serum (GIBCO). The K562 erythroleukemia cell line and Yac-1 cell line were grown in Rosewell Park Memorial Institute (RPMI)-1640 (GIBCO) medium containing $10 \%$ fetal bovine serum.

\footnotetext{
Animals

Six-week-old specific pathogenic-free BALB/c male mice were purchased from the Dae-Han BioLink (DBL), Chungbuk, Korea. Mice were maintained in the Laboratory of Animal Experiment, Institute of Bioscience and Technology, Handong Global University. They were maintained $23 \pm 2{ }^{\circ} \mathrm{C}$ with $55 \% \pm$ $20 \%$ relative humidity and 12 hour day and night cycle. Water and diets were supplied ad libitum. Each group was composed of 5 mice, and KMLC was administered intraperitoneally (200 ng/head or $500 \mathrm{ng} / \mathrm{head}$ ) as well as a Phosphate Buffered Saline (PBS) control as vehicle control 24 hours before sacrifice for analysis of granzyme-B and perforin expression in splenocytes. Freshly isolated splenocytes were washed in cold PBS twice and passed through a $40-\mu \mathrm{m}$ cell strainer (Corning).
}

After centrifugation at $400 \times \mathrm{x}$ for 10 minutes, erythrocytes were eliminated by red blood cell (RBC) specific lysis buffer (Calbiochem) 5 minute in room temperature. After washing once again, the collected pellet was resuspended in $40 \mathrm{~mL}$ cold PBS, then used intracellular staining after counts with a hemocytometer.

\section{Extraction of KMLC}

KMLC was prepared as described previously. ${ }^{10}$ Briefly, the chopped leaves of KM were homogenized in 10 volumes (weight/volume) of distilled water for 30 seconds. The homogenates were then stirred overnight at $4{ }^{\circ} \mathrm{C}$. The insoluble materials were removed by centrifugation at $15,000 \mathrm{x} g$ for 30 minutes at $4{ }^{\circ} \mathrm{C}$, and the supernatant was passed through a $0.45-\mu \mathrm{m}$ filter (Millipore, Massachusetts, USA) followed by a $0.2-\mu \mathrm{m}$ filter (Millipore, Massachusetts, USA). The final filtered supernatant was loaded onto a Sepharose 4B column (GE Healthcare, Sweden), which had been treated with $0.2 \mathrm{M}$ $\mathrm{HCl}$ for 2.5 hours. The bound material (KMLC) was eluted using lactose buffer $(0.1 \mathrm{M}$ lactose in $0.01 \mathrm{M}$ sodium phosphate and $0.14 \mathrm{M} \mathrm{NaCl}, \mathrm{pH}$ 7.3). The fractions containing KMLC were pooled, dialyzed against distilled water, and freeze-dried. For mouse experiments, KMLC was dissolved in PBS, the concentration was measured with a bicinchoninic acid (BCA) protein assay kit (Thermo Scientific), and stored at $4{ }^{\circ} \mathrm{C}$ prior to use.

\section{Preparation of KMLC B chain}

The separation of the KMLC B chain has been reported previously. ${ }^{24}$ In brief, KMLC was dissolved in PBS with 5\% $\beta$-mercaptoethanol (Sigma, Missouri, USA) and incubated overnight at $25{ }^{\circ} \mathrm{C}$. It was then loaded onto a lactose affinity column (Sigma, Missouri, USA) that had previously been equilibrated with the same buffer. The unbound fraction was eluted with the same buffer, and the bound fraction was eluted with PBS containing 0.1 M lactose (Sigma, Missouri, USA). The fractions containing the B chain were then pooled, dialyzed against distilled water, and freeze-dried.

\section{$B A T D A$ release cytotoxicity assay}

The NK cell cytotoxicity assay was performed as described previously. ${ }^{25}$ Briefly the target cells, the human leukemia K562 and Yac-1 cell lines, were labeled with BATDA complex (Thermo Fisher) and co-cultured with the NK92 cell line or with splenocytes as effector cells. The target cells $\left(5 \times 10^{3}\right.$ cells/ well) were cultured in round-bottomed microwell plates with various concentrations of effector cells in duplicate under standard culture conditions for 4 hours. Spontaneous BATDA release wells were composed of target cells and $100 \mu \mathrm{L}$ of normal medium and the maximum release wells contained target cells with $100 \mu \mathrm{L}$ of Triton X-100 lysis buffer. After the 4-hour incubation, $20 \mu \mathrm{L}$ of the supernatant was transferred to a flat-bottomed plate and Europium solution was added. Finally, fluorescence was measured in a time-resolved fluorometer (PerkinElmer VICTOR 2D). To check reproducibility, all experiments were performed at least 3 times.

The percentage of specific lysis was calculated using the following formula: 
specific BATDA release $=($ experimental count - spontaneous count/maximum count - spontaneous count $) \times 100$.

\section{Intracellular staining and flow cytometry}

NK92 cells were stained with antibodies detecting granzyme- $B$ and perforin, and splenocytes were stained for surface CD3 and NK1.1 and intracellular granzyme-B and perforin, as described. Fluorescein isothiocyanate (FITC)-conjugated anti-mouse $\mathrm{CD} 3$ monoclonal antibody $(\mathrm{mAb})(\mathrm{BD}$, California, USA), FITC-conjugated anti-human Gzmb mAb (BD), Phycoerythrin (PE)-conjugated anti-human Prf mAb (BD), PE -conjugated anti-mouse Gzmb mAb (BD), and PE-conjugated anti-mouse $\operatorname{Prf} \mathrm{mAb}$ (e-Bioscience) were used. Samples were then acquired on a flow cytometer and analyzed. Isotype controls were used to set quadrant gates. In a separate study, NK92 cells were co-cultured in 6-well culture plates (Falcon) with various concentrations of KMLC or B chain for a range of time periods. After 24 hours, NK92 cells were stained for surface NKp44 and NKG2D using specific mAbs (Biolegend, California, USA) conjugated with FITC and PE. Samples were acquired on a flow cytometer (Beckman Coulter, California, USA) and analyzed. To check reproducibility, all experiments were performed at least 3 times.

\section{Preparation of protein lysates and immunoblots}

Protein lysates were prepared from KMLC-activated spleen and NK92 cell lines using a lysis buffer (Intron, South Korea). Proteins were separated by sodium dodecyl sulfate polyacrylamide gel electrophoresis (SDS-PAGE) and transferred to nitrocellulose membranes (Millipore, Massachusetts, USA). Activation of MAPK was detected using p-Extracellular signal -Regulated Kinase (ERK) (\#4370, Cell signaling, Massachusetts, USA) and p-JNK (\#9255, Cell signaling, Massachusetts, USA) mAbs diluted 1:1000.

\section{Results}

Effect of KMLC on NK92 cell and splenocyte cytotoxicity

To explore the effect of KMLC on NK cell activity, we examined whether KMLC enhanced NK92 cell and splenocyte cytotoxicity. Initially, we determined the direct cytotoxicity of a range of KMLC concentrations up to $200 \mathrm{ng} / \mathrm{mL}$ on NK92 cells. No significant effect of KMLC on viability of the NK92 cell line was observed at concentrations below $200 \mathrm{ng} / \mathrm{mL}$ (Figure 1A). The NK cell-mediated cytotoxicity assay was performed using K562 as the target cells for NK92 cells and Yac-1 cells for splenocytes. The NK92 cells primed with 10-100 $\mu \mathrm{g} / \mathrm{mL}$ of KMLC were co-incubated 4 hours with the target cells at the indicated effector cell to target cell ratio (E:T ratio). As shown in Figure 1B and 1C, the cytolytic activities of both NK92 cells and splenocytes were enhanced by treatment with KMLC in a dose-dependent manner. At a KMLC concentration of $100 \mathrm{ng} / \mathrm{mL}$, activity was increased by $40 \%$ compared with that of the untreated cells at the same E:T ratio (25:1) in NK92 cells (Figure 1B). In addition, treatment of splenocytes with 100 $\mathrm{ng} / \mathrm{mL}$ KMLC produced a $25 \%$ increase in cytotoxic activity compared with that of the untreated cells at the same E:T ratio (250:1; Figure 1C).
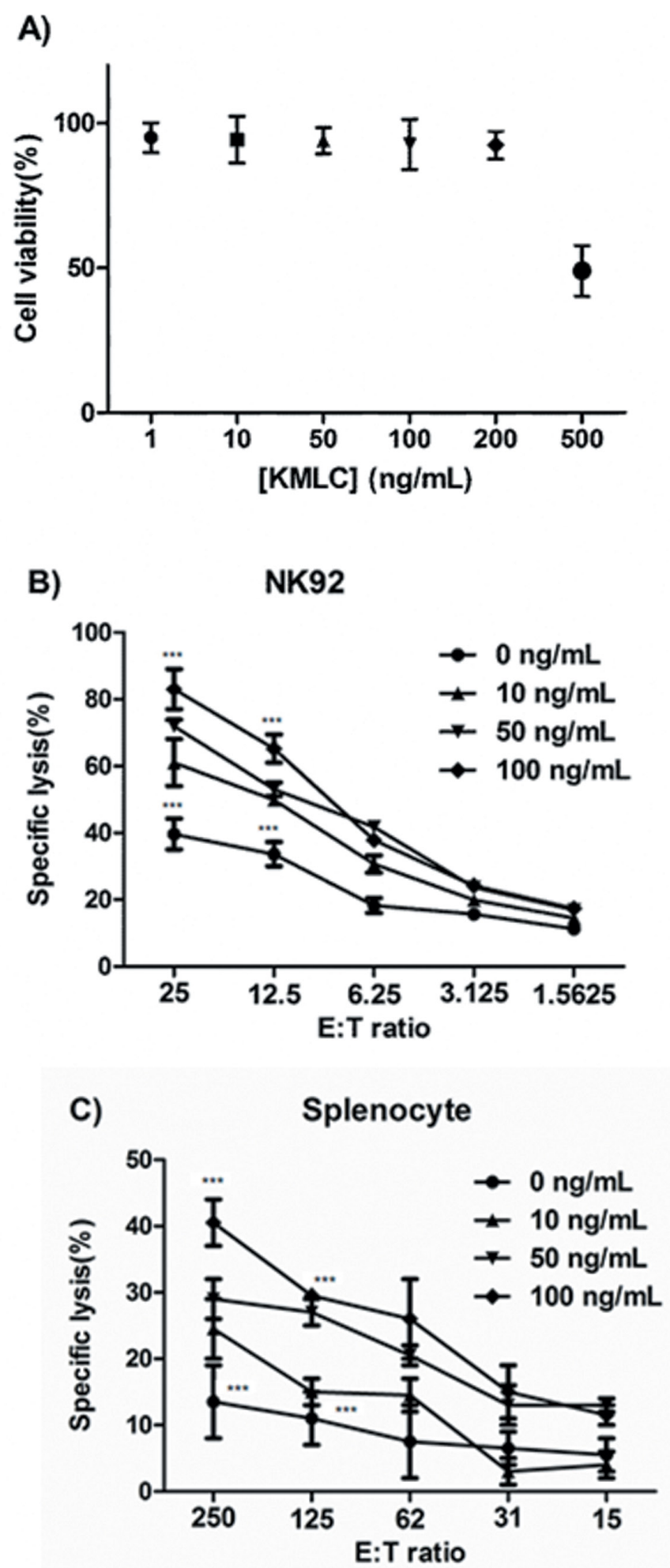

Figure 1. KMLC enhanced NK cell cytotoxic activity. (A) Viability of NK92 cells treated with KMLC. NK92 cells (B) or splenocytes $(\mathrm{C})$ were stimulated with the indicated concentrations of KMLC and co-cultured with K562 cells for $4 \mathrm{~h}$ at the indicated E:T ratios. Cytotoxic activity was performed by BATDA release assay. 


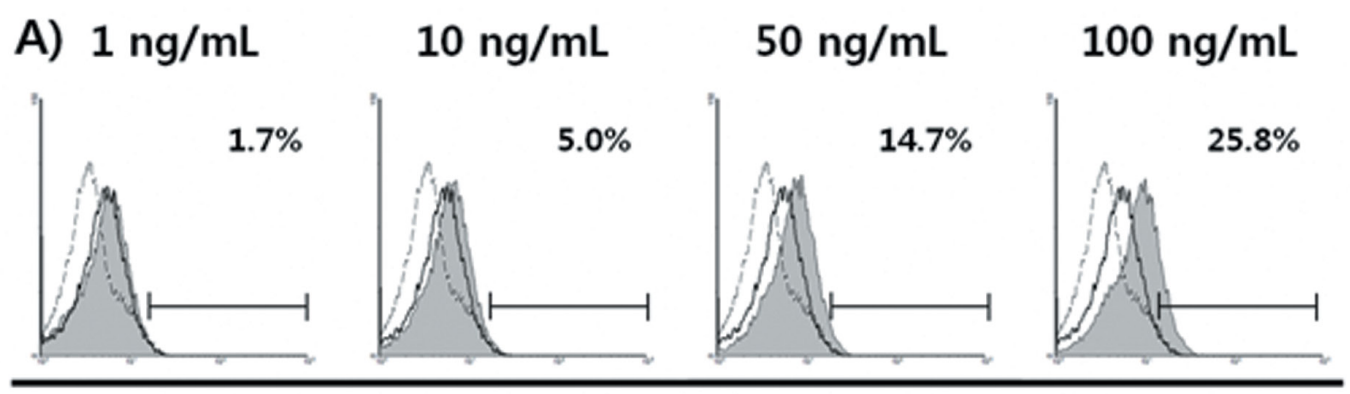

Perforin

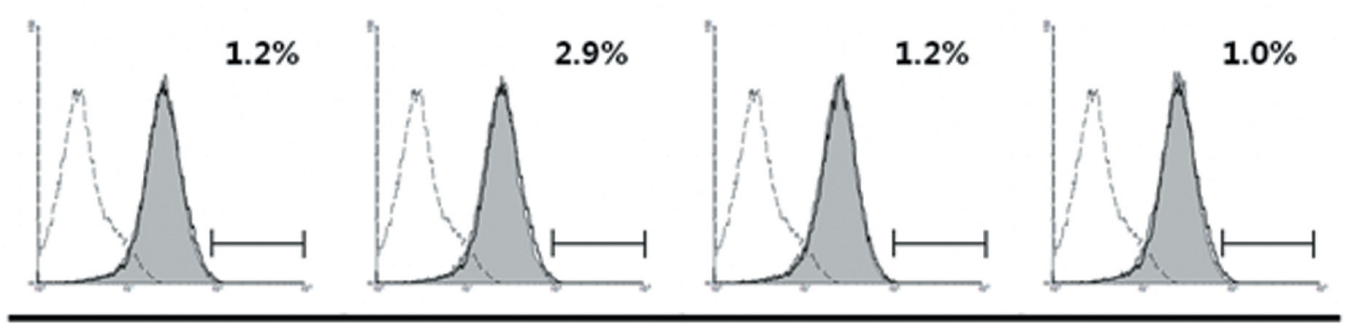

Granzyme b
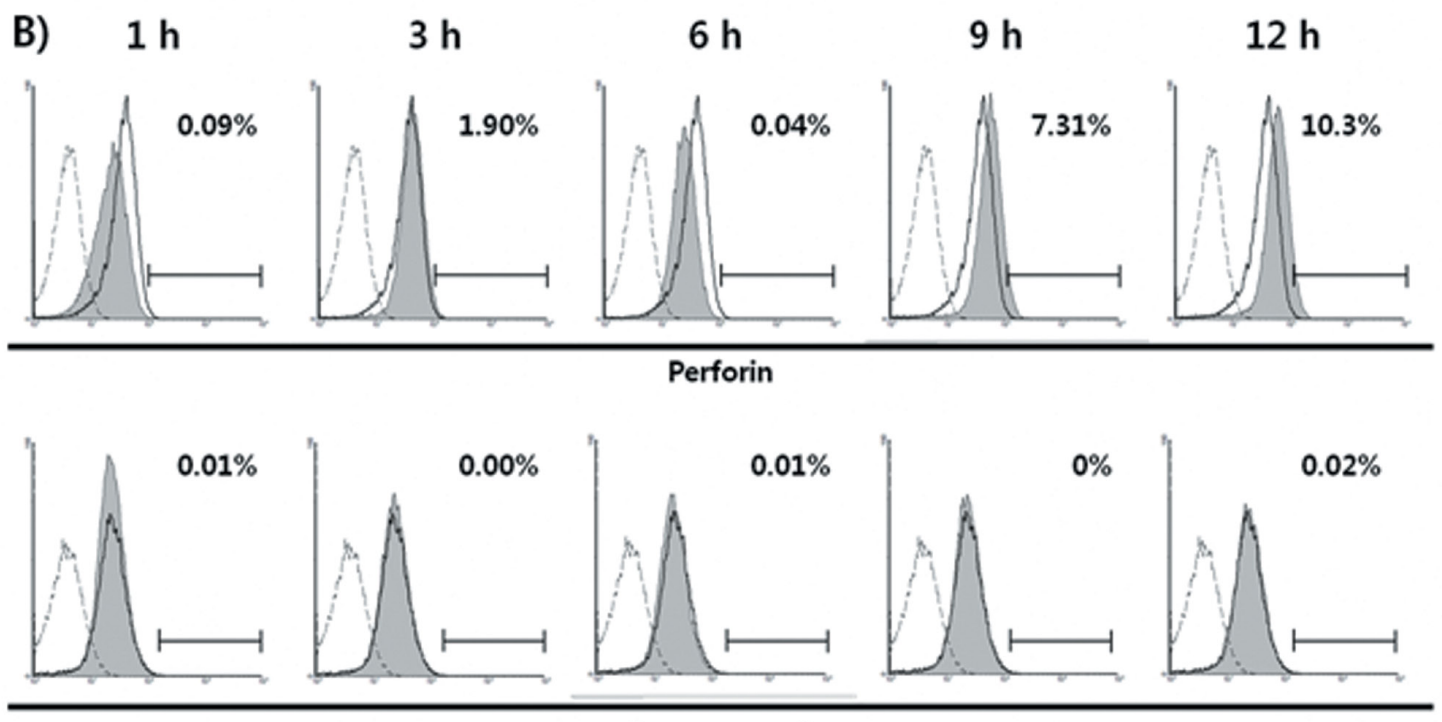

Granzyme b

Figure 2. KMLC increased perforin expression in NK92 cells. $1 \times 10^{6}$ NK92 cells were treated with KMLC using the indicated doses and time periods. Representative flow cytometry histogram plots were shown for perforin and granzyme-B expression. The expression of granzyme-B (upper panels) and perforin (bottom panels) is shown at a range of KMLC concentrations (A) and times (B). Dash lines and bold lines indicate non-treatment and isotype control staining of the cells. Values of the fold increase with respect to isotype control are reported.

\section{Expression of perforin and granzyme-B in the NK92 cell line}

$\mathrm{NK}$ and $\mathrm{T}$ cell cytotoxicity is mediated by expression of cytolytic granules containing granzyme-B and perforin. ${ }^{16}$ Therefore, we examined the effect of KMLC on perforin and granzyme-B expression in the NK92 cell line. NK92 cells were primed with KMLC at various concentrations for a range of time periods, and the expression of perforin and granzyme-B was assessed by flow cytometry. NK92 cells were also pretreated to examine time-dependent effects. As shown in Figure 2, KMLC treatment increased perforin expression in a dose- and time-dependent manner. At a KMLC concentration of $100 \mathrm{ng} / \mathrm{mL}$, the perforin expression level was increased by $25 \%$ compared with that observed in the untreated cells. However, no significant upregulation of granzyme-B was detected in KMLC-treated cells (Figure 2A and 2B).

\section{Expression of perforin and granzyme-B in splenocytes}

To explore whether in vivo treatment with KMLC also induced perforin expression, we assessed perforin expression in splenocytes of mice injected with KMLC. Intraperitoneal injection of KMLC enhanced the perforin-containing $\mathrm{NK}$ cell population $\left(\mathrm{CD} 3-\mathrm{NK} 1.1^{+} \mathrm{Prf}^{+}\right)$in splenocytes in a 
A)
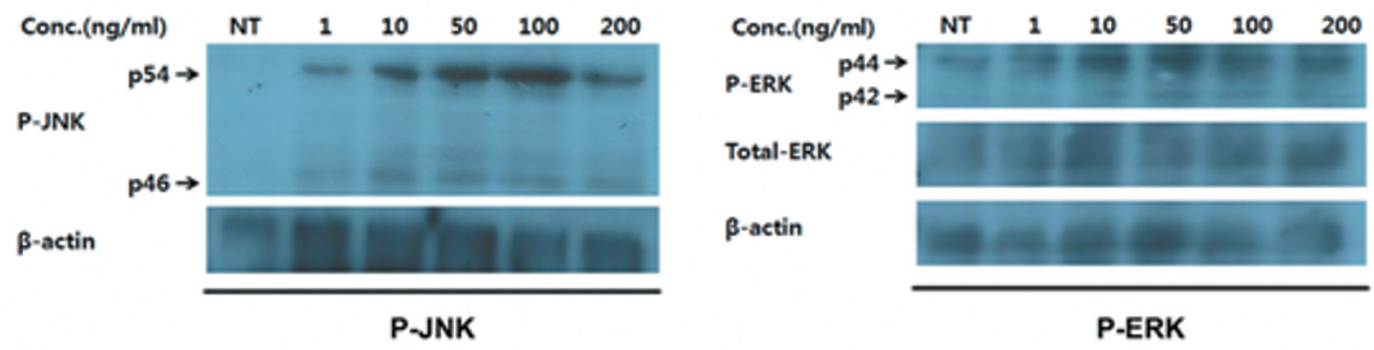

B)
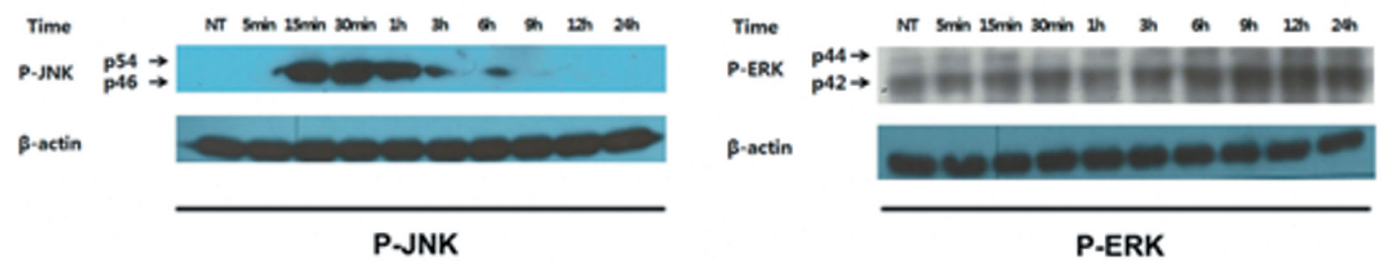

C)
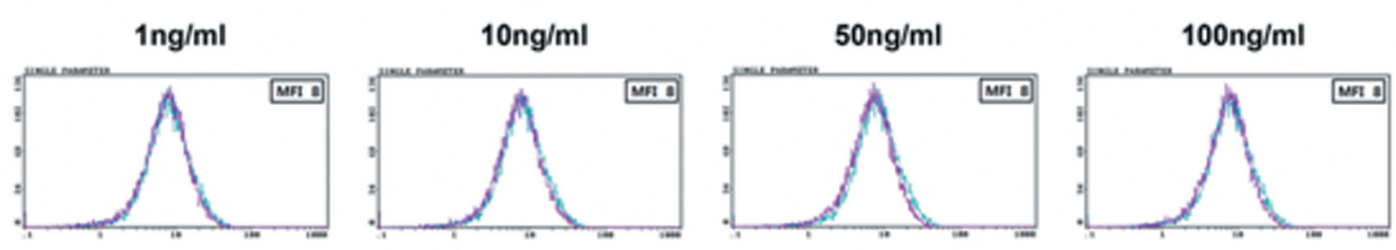

Perforin

D)

NK92

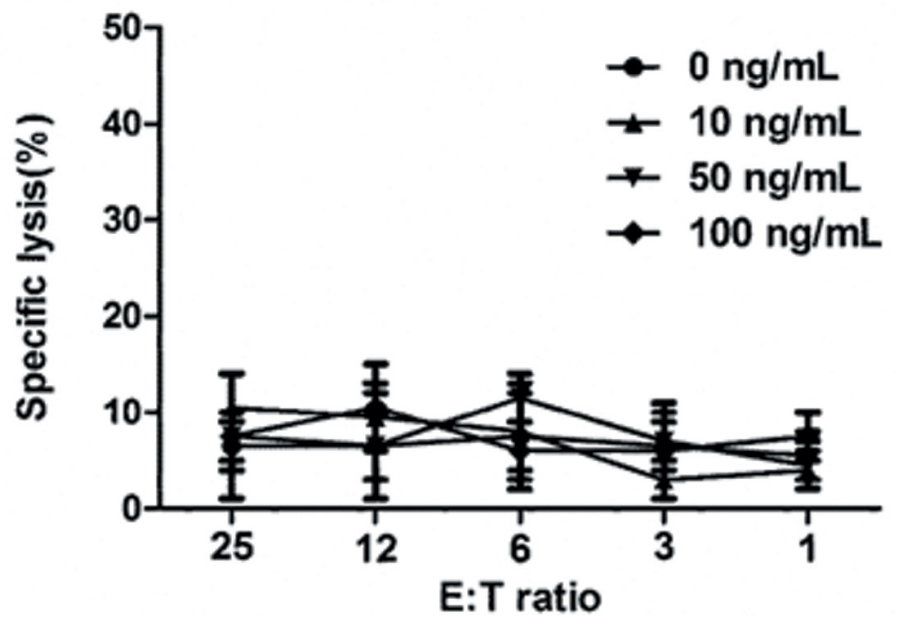

Figure 3. KMLC enhanced perforin expression via the MAPK pathway. $1 \times 10^{6} \mathrm{NK} 92$ cells were pretreated with KMLC at the indicated doses and times. (A, B) Phosphorylated JNK and ERK were detected by western blot. $\beta$-actin was used as the loading control. (C) The effect of SP600125 (20 nM) on perforin expression of NK92 cells. (D) BATDA release assay was performed with treatment of 20nM SP600125 to NK92 cell line against K562 target cells.

dose-dependent manner. However, no significant upregulation of granzyme-B was detected following KMLC treatment.

\section{KMLC upregulated phosphorylation of JNK and ERK}

MAPK signaling has been shown to be associated with NK cell cytotoxicity. ${ }^{22}$ Therefore, we assessed whether KMLC activated the MAPK signaling pathway in the NK92 cells.
MAPK phosphorylation was examined following treatment with KMLC at various concentrations for a range of time periods. As shown in Figure $\mathbf{3 A}$ and $\mathbf{3 B}$, KMLC treatment increased the level of phosphorylated JNK in 30 minutes. However, altered ERK1/2 phosphorylation was not detected following KMLC treatment. To address whether the activity of KMLC on NK cytotoxicity and perforin expression was 
dependent on JNK activation, SP600125, a JNK inhibitor, was added to the NK92 cell line prior to measuring their cytotoxic activity towards target cells and their perforin expression. As shown in Figure 3C, no difference in perforin expression was detected in cells exposed to various concentrations of KMLC, and NK cell cytotoxicity against K562 target cells was decreased by KMLC treatment (Figure 3D). These data suggested that JNK must be a crucial kinase involved in the regulation of NK

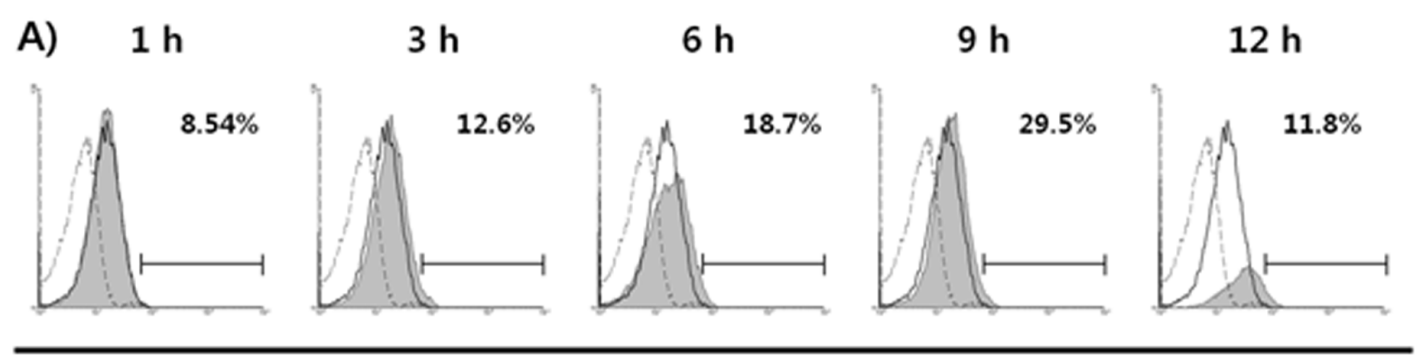

NKG2D
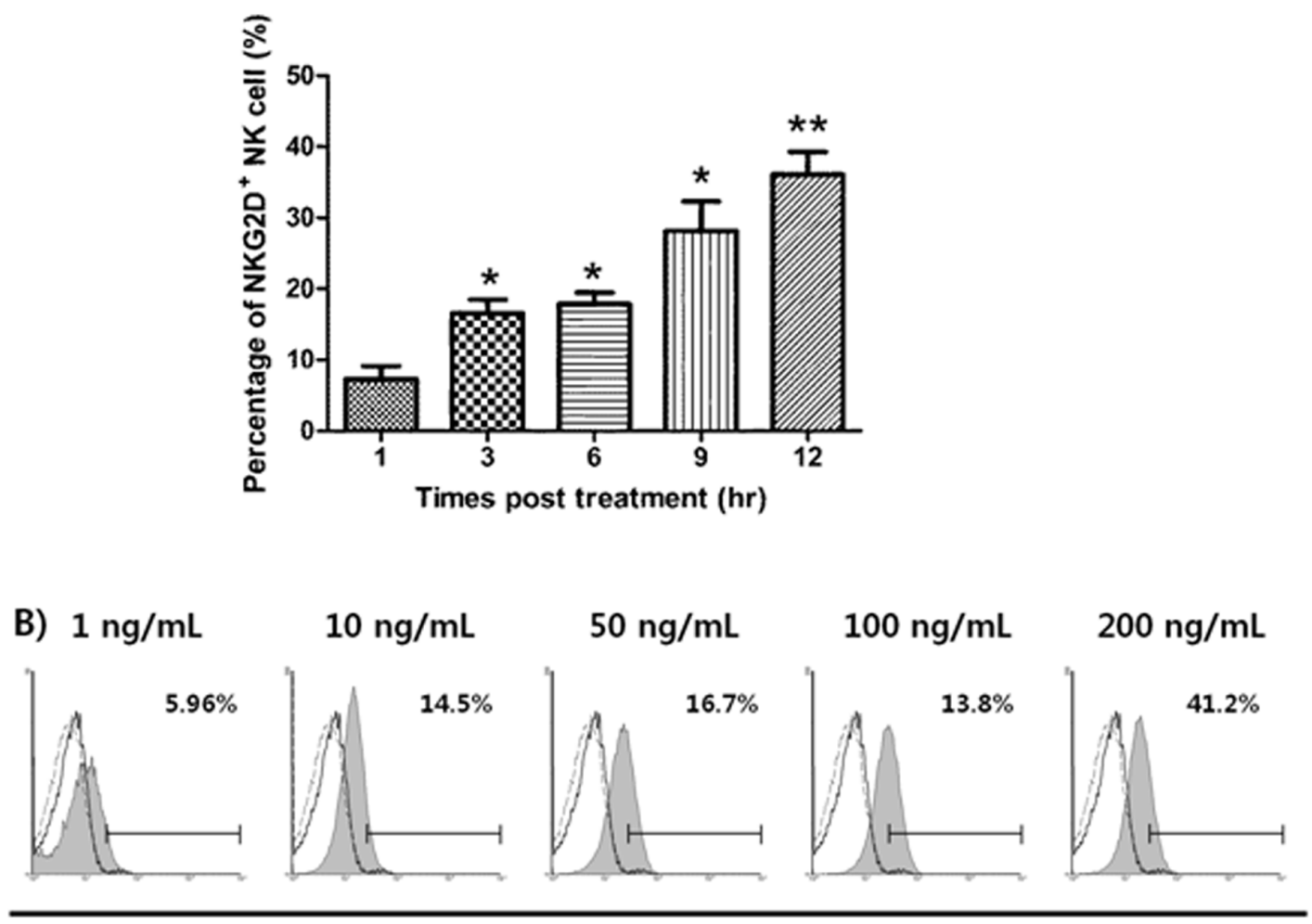

\section{NKG2D}

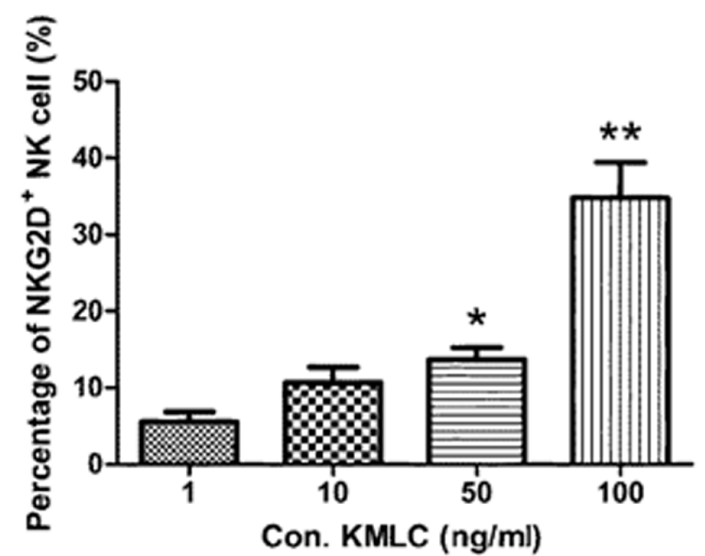

Figure 4. NKG2D receptor expression in NK92 cells was increased by KMLC. The levels of NKG2D and NKp44 were analyzed by flow cytometry. Representative flow cytometry histogram plots showing the fluorescence intensity of the FITC-conjugated anti-NKG2D monoclonal antibody (A and B) and PE-conjugated anti-NKp44 monoclonal antibody (C and D), compared with isotype controls. Values of the respective MFI fold increase are reported. ${ }^{\star} \mathrm{P}<0.05$. 
C) $1 \mathrm{~h}$

$3 \mathrm{~h}$

$6 \mathrm{~h}$

$9 \mathrm{~h}$

$12 \mathrm{~h}$

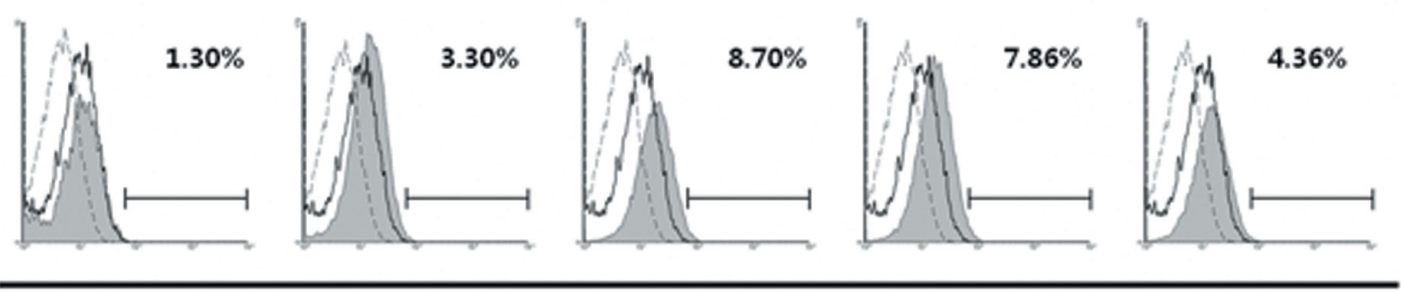

NKp44

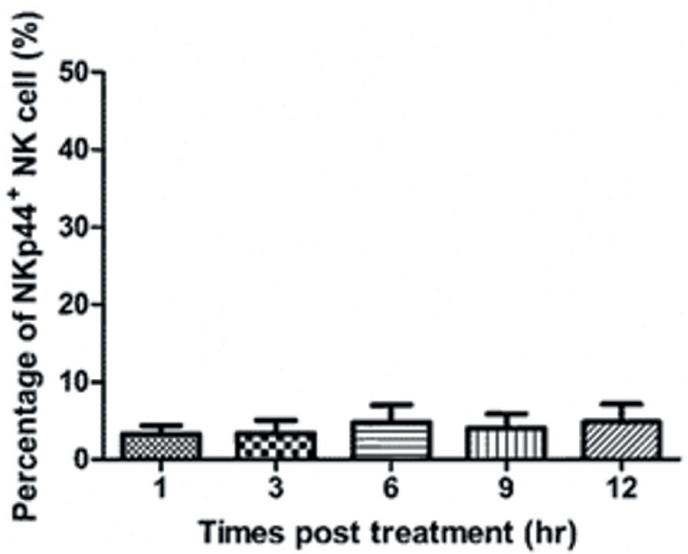

D) $1 \mathrm{ng} / \mathrm{mL}$

$10 \mathrm{ng} / \mathrm{mL}$

$50 \mathrm{ng} / \mathrm{mL}$

$100 \mathrm{ng} / \mathrm{mL}$

$200 \mathrm{ng} / \mathrm{mL}$
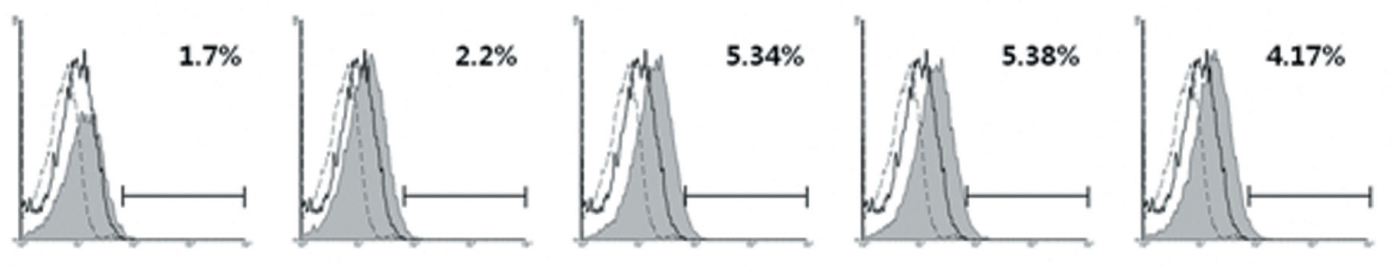

NKp44

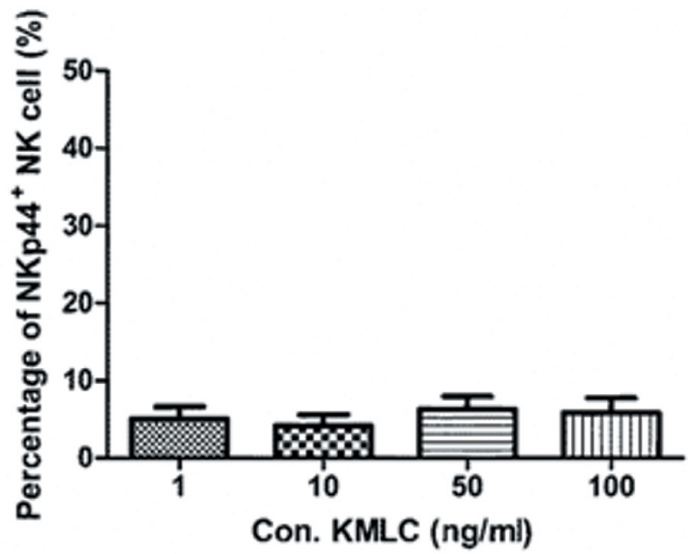

Figure 4. (Continued) NKG2D receptor expression in NK92 cells was increased by KMLC.

cell cytotoxicity via perforin expression.

KMLC upregulated the expression of NKG2D and NKp44 in the NK92 cell line

NKG2D is a C-type lectin-like receptor and its activation triggers perforin expression and NK cell cytotoxicity. ${ }^{26} \mathrm{NKp} 44$ is a human natural cytotoxicity receptor (NCR) family receptor classified as part of the immunoglobulin superfamily, which is composed of NKp44, NKp46, and NKp30. ${ }^{27}$ These receptors have been reported to be upstream of the MAPK pathway, ${ }^{28}$ and we, therefore, examined whether KMLC enhanced the expression of NKG2D or NKp44. KMLC treatment enhanced the expression of NKG2D in a dose- and time-dependent manner (Figure 4A and 4B). KMLC enhanced NKG2D expression 
A

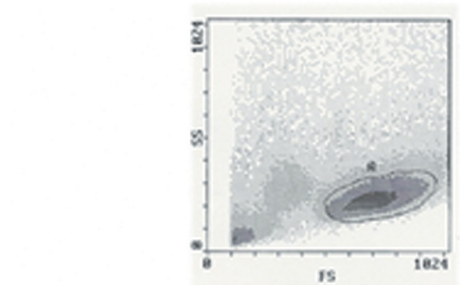

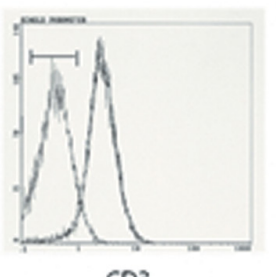

$\mathrm{CO} 3$

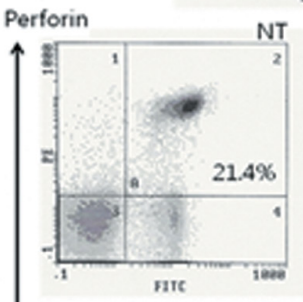

NT
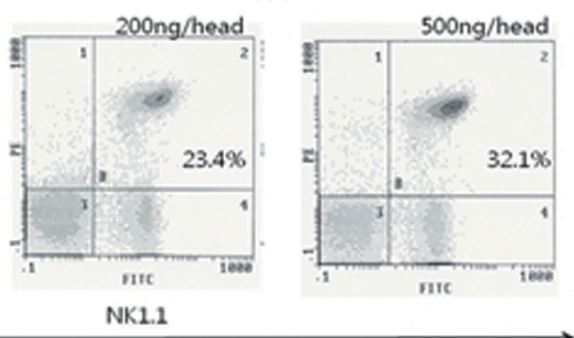

B
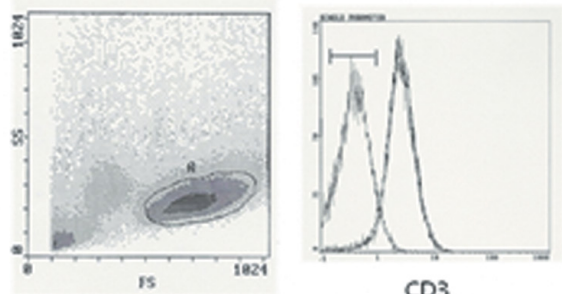

CD3
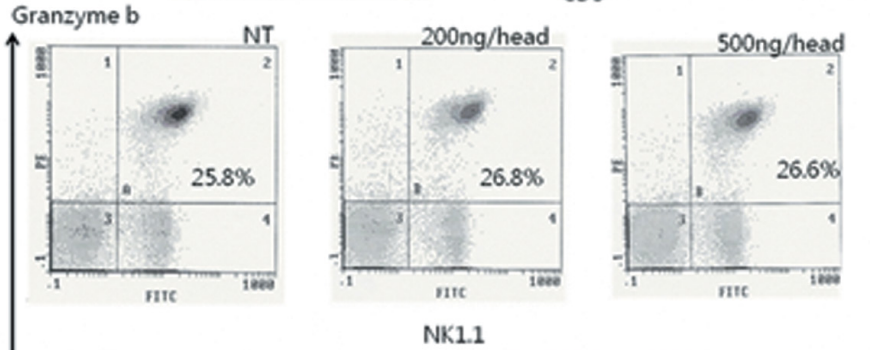

Figure S1. KMLC upregulated perforin expression in splenocyte. After BALB/c mice were injected with a range of KMLC concentrations, the expression of perforin and granzyme-B were analyzed in their splenocytes; (A) \% population of perforin-positive cells in splenocytes; (B) \% populaion of granzyme-B-positive cells in splenocytes.

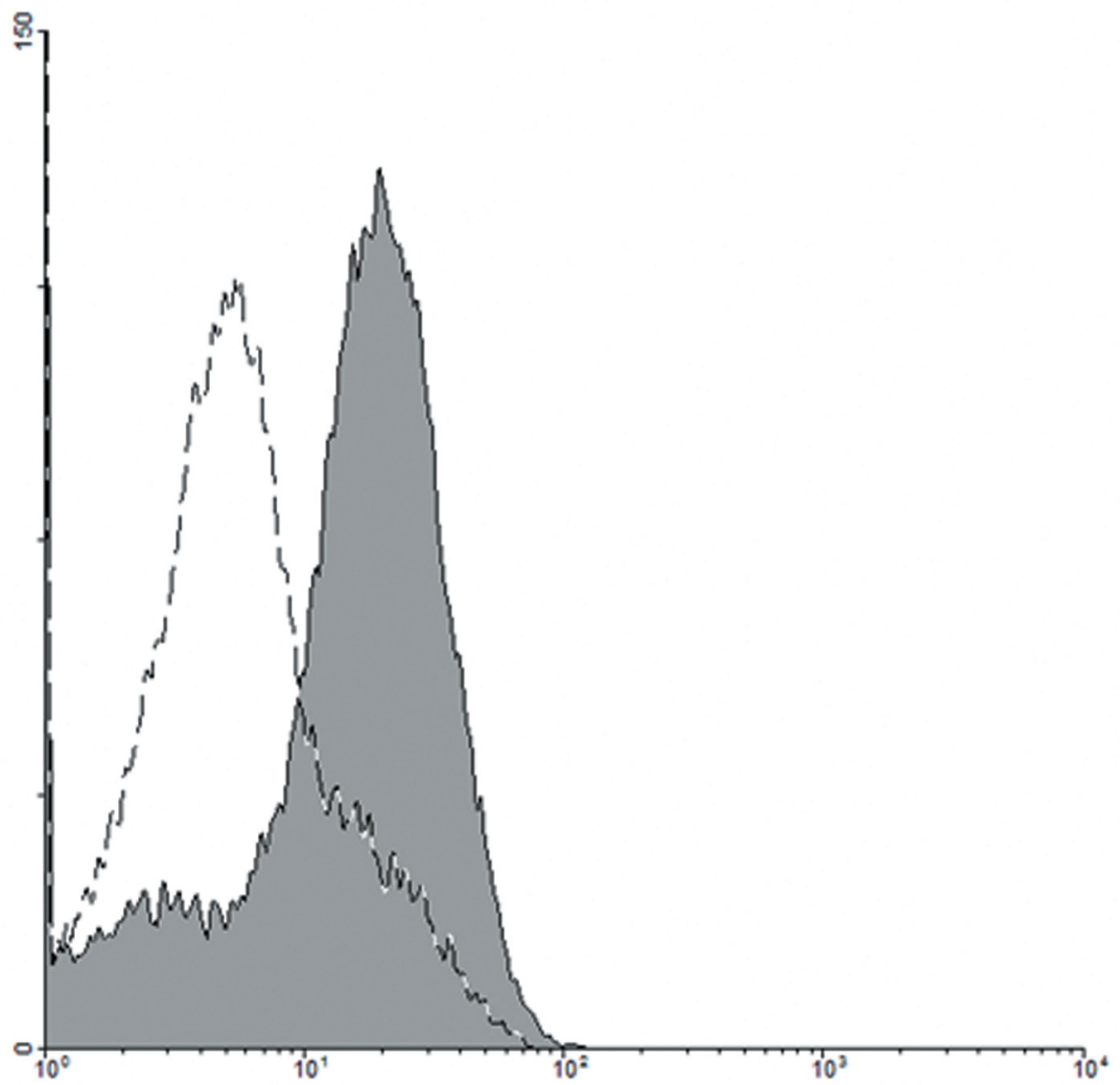

Figure S2. KMLC B chain increased perforin expression in NK92 cells. $1 \times 10^{6}$ NK92 cells were treated with KMLC B chain. Representative flow cytometry histogram plot was shown for perforin expression. The expression of perforin is shown at $20 \mathrm{ng} / \mathrm{mL}$ of $\mathrm{B}$ chain concentration. Dash line indicates non-treatment and filled area is a sample-treated cells.

by more than $20 \%$ at 9 hours and showed a $35 \%$ increase in expression in the $200 \mathrm{ng} / \mathrm{mL}$-treated group compared with the untreated group. However, there was no effect on NKp44 expression. These data suggested that KMLC had a potential role as a NK cell enhancer via modulation of expression of the NKG2D surface receptor, but not of the NKp44 NCR family.
Expression of perforin by KMLC B chain treatment in the NK92 cell line

KMLC B chain is a subchain of KMLC, and it has immunoadjuvant activity with low cytotoxicity. ${ }^{24}$ Therefore, we examined the effect of KMLC B chain on perforin expression in the NK92 cell line. NK92 cells were primed with KMLC B 
chain, and the expression of perforin was assessed by flow cytometry. As shown in Figure S2, treatment with KMLC B chain increases perforin expression (Figure S2).

At a KMLC concentration of $100 \mathrm{ng} / \mathrm{mL}$, the perforin expression level was increased by $25 \%$ compared to that observed in the untreated cells. However, no significant upregulation of granzyme-B was detected in KMLC-treated cells (Figure 2A and 2B).

\section{Discussion}

The investigation of cancer treatments involving improvement of host defense systems is a novel approach. ${ }^{29}$ The important immune cell in host defense is the NK cell, which kills tumor cells and virus-infected cells. ${ }^{16} \mathrm{NK}$ cell therapy is an innovative and well- researched method. Normally, IL-2 is used for activation of $\mathrm{NK}$ or $\mathrm{T}$ cells isolated from cancer patients. ${ }^{30}$ It improves their killing effects and helps to treat the illness. However, this therapy has limitations for the patient, because of the side effects of IL-2. To solve this problem, scientists have aimed to discover new natural compounds with fewer side effects. ${ }^{21}$ In a previous study, we showed that natural KM extract stimulated NK cell-mediated cytotoxicity in a mouse model. ${ }^{8}$ This finding showed that KM extract might represent a potential natural compound for cancer treatment.

The present study examined the mechanisms of NK cell cytotoxicity involving the activating receptor and signaling molecules downstream of the NKG2D receptor. KMLC appeared to activate NKG2D, leading to the activation of JNK phosphorylation. Finally, this resulted in upregulation of perforin expression, which improved the targeting of cancer cells.

In this study, we clearly showed that KMLC promoted perforin-mediated NK cell cytotoxicity via increased MAPK pathway (JNK) phosphorylation. KMLC enhanced NK92 cell cytotoxicity against the K562 human leukemia cell line, which is major histocompatibility complex (MHC) class I-deleted. The cytotoxicity was measured by a BATDA release assay that is a more specific and rapid method than the traditional ${ }^{51}$ Chromium $(\mathrm{Cr})$ release assay.

Interestingly, only NKG2D was upregulated when co-cultured with KMLC in vitro. ${ }^{26}$ The most effective NK cell activating receptor, NKG2D, is closely related to $\mathrm{NK}$ cell cytotoxicity against cancer and virus-infected cells. However, no difference was observed in the expression of the NKp44 receptor, which is classified as a member of the Ig superfamily, along with NKp44, NKp46, and NKp30. ${ }^{27}$ This may be due to the NCR receptor binding affinity for viral glycoproteins. ${ }^{31}$ In addition, perforin expression was increased following exposure to KMLC. Resveratrol also upregulates perforin protein in NK cells and activates the MAPK pathway by NKG2D activation. ${ }^{21}$ Although the phenomenon results in demonstrable cytotoxicity in mice and cell lines, the signaling and downstream mechanisms involved in NK cell cytotoxicity are still unclear. We will approach that which is the NK cell surface receptor with KMLC, binding affinity, and other intracellular signal molecules of NK cells upstream of JNK.

KMLC is composed of A and B subchains. ${ }^{7}$ The B chain binds to carbohydrates on the cell surface receptor, and the A chain hinders translation and induces apoptosis as a ribosome-inactivating protein (RIP). ${ }^{7}$ In previous studies, the $\mathrm{B}$ chain was shown to have a variety of immunological activities with low cytotoxicity. ${ }^{32}$ We isolated the B chain and examined its immunomodulatory activity on $\mathrm{NK}$ cell cytotoxicity. These findings indicated that the B chain was the major active compound involved in modulation of NK cell cytotoxicity. Future studies will aim to identify the optimal mixture and concentration of KMLC and B chain for the development of a new natural anti-cancer drug.

\section{Conflict of interest}

The authors declare that there is no conflict of interest regarding the publication of this paper.

\section{Acknowledgments}

This research was supported by No. 20120048 of Handong Global University Research Grants. This grant does not lead to any conflict of interests regarding the publication of this paper.

\section{Ethics approval}

All of the animal experiments were approved by the Ethics Review Committee of the Handong Global University.

\section{References}

1. Lavelle EC, Grant G, Pusztai A, Pfuller U, O’Hagan DT. Mucosal immunogenicity of plant lectins in mice. Immunology. 2000;99:30-7.

2. Gabius HJ. Probing the cons and pros of lectin-induced immunomodulation: case studies for the mistletoe lectin and galectin-1. Biochimie. 2001;83:659-66.

3. Bloksma N, Van Dijk H, Korst P, Willers JM. Cellular and humoral adjuvant activity of mistletoe extract. Immunobiology. 1979;156:309-18.

4. Kuttan G, Kuttan R. Immunological mechanism of action of the tumor reducing peptide from mistletoe extract (NSC 635089) cellular proliferation. Cancer Lett. 1992;66:123-30.

5. Kuttan G, Vasudevan DM, Kuttan R. Effect of a preparation from Viscum album on tumor development in vitro and in mice. J Ethnopharmacol. 1990;29:35-41.

6. Metzner G, Franz H, Kindt A, Fahlbusch B, Suss J. The in vitro activity of lectin I from mistletoe (ML I) and its isolated A and B chains on functions of macrophages and polymorphonuclear cells. Immunobiology. 1985;169:461-71.

7. Franz H. Mistletoe lectins and their A and B chains. Oncology. 1986;43 Suppl 1:23-34.

8. Yoon TJ, Yoo YC, Kang TB, Baek YJ, Huh CS, Song SK, et al. Prophylactic effect of Korean mistletoe (Viscum album coloratum) extract on tumor metastasis is mediated by enhancement of $\mathrm{NK}$ cell activity. Int J Immunopharmacol. 1998;20:163-72.

9. Khwaja TA, Varven JC, Pentecost S, Pande H. Isolation of biologically active alkaloids from Korean mistletoe Viscum album, coloratum. Experientia. 1980;36:599-600.

10. Ye W, Nanga RP, Kang CB, Song JH, Song SK, Yoon HS. Molecular Characterization of the recombinant A-chain of a type II RIP from Viscum album coloratum and structural basis on it ribosome-inactivating activity and the sugar-binding properties of B-chain. J Biochme Mol Biol. 2006;39:560-70.

11. Khil LY, Kim W, Lyu S, Park WB, Yoon JW, Jun HS. Mechanisms involved in Korean mistletoe lectin-induced apoptosis of cancer cells. World J Gastroenterol. 2007;13:2811-8.

12. Lyu SY, Park WB. Effects of Korean mistletoe lectin (Viscum album coloratum) on proliferation and cytokine expression in human peripheral blood mononuclear cells and T-lymphocytes. Arch Pharm Res. 2007;30: 1252-64.

13. Park HJ, Hong JH, Kwon HJ, Kim Y, Lee KH, Kim JB, et al. TLR4-mediated activation of mouse macrophages by Korean mistletoe lectin-C (KML-C) Biochem Biophys Res Commun. 2010;396:721-5. 
14. Yoon TJ, Yoo YC, Kang TB, Song SK, Lee KB, Her E, et al. Antitumor activity of the Korean mistletoe lectin is attributed to activation of macrophages and NK cells. Arch Pharm Res. 2003;26:861-7.

15. Park CH, Lee DW, Kang TB, Lee KH, Yoon TJ, Kim JB, et al. cDNA cloning and sequence analysis of the lectin genes of the Korean mistletoe (Viscum album coloratum). Mol Cells. 2001;12:215-20.

16. Vivier E, Tomasello E, Baratin M, Walzer T, Ugolini S. Functions of natural killer cells. Nat Immunol. 2008;9:503-10.

17. Williams NS, Klem J, Puzanov IJ, Sivakumar PV, Bennett M, Kumar V. Differentiation of NK1.1+, Ly49+ NK cells from flt3+ multipotent marrow progenitor cells. J Immunol. 1999;163:2648-56.

18. Eller MA, Koehler RN, Kijak GH, Eller LA, Guwatudde D, Marovich MA, et al. Human immunodeficiency virus type 1 infection is associated with increased NK cell polyfunctionality and higher levels of KIR3DL1+ NK cells in ugandans carrying the HLA-B Bw4 motif. J Virol. 2011;85:4802-11.

19. Le Bouteiller P, Siewiera J, Casart Y, Aguerre-Girr M, El Costa H, Berrebi A, et al. The human decidual NK-cell response to virus infection: what can we learn from circulating NK lymphocytes? J Reprod Immunol. 2011;88:170-5.

20. Fehniger TA, Cai SF, Cao X, Bredemeyer AJ, Presti RM, French AR, et al. Acquisition of murine $\mathrm{NK}$ cell cytotoxicity requires the translation of a pre-existing pool of granzyme B and perforin mRNAs. Immunity. 2007;26:798-811.

21. Lu CC, Chen JK. Resveratrol enhances perforin expression and NK cell cytotoxicity through NKG2D-dependent pathways. J Cell Physiol. 2010;223:343-51.

22. Vivier E, Nunes JA, Vely F. Natural killer cell signaling pathways. Science. 2004;306:1517-9.

23. Blomberg K, Hautala R, Lovgren J, Mukkala VM, Lindqvist C, Akerman $\mathrm{K}$. Time-resolved fluorometric assay for natural killer activity using target cells labelled with a fluorescence enhancing ligand. J Immunol Methods. 1996;193:199-206.
24. S.M. Her HA, K.D. Kim, Y.H. Kim, T.J. Yoon, J.B. Kim. Immunoadjuvant Activity of Korean Mistletoe Lectin B-chain. Kor J Pharmacogn. 2011;42: 246-52.

25. von Zons P, Crowley-Nowick P, Friberg D, Bell M, Koldovsky U, Whiteside TL. Comparison of europium and chromium release assays: cytotoxicity in healthy individuals and patients with cervical carcinoma. Clin Diagn Lab Immunol. 1997;4:202-7.

26. McFarland BJ, Kortemme T, Yu SF, Baker D, Strong RK. Symmetry recognizing asymmetry: analysis of the interactions between the C-type lectin-like immunoreceptor NKG2D and MHC class I-like ligands. Structure. 2003;11:411-22.

27. Cantoni C, Ponassi M, Biassoni R, Conte R, Spallarossa A, Moretta A, et al. The three-dimensional structure of the human NK cell receptor NKp44, a triggering partner in natural cytotoxicity. Structure. 2003;11:725-34.

28. Li C, Ge B, Nicotra M, Stern JN, Kopcow HD, Chen X, et al. JNK MAP kinase activation is required for MTOC and granule polarization in NKG2D-mediated NK cell cytotoxicity. Proc Natl Acad Sci U S A. 2008;105: 3017-22.

29. Salagianni M, Lekka E, Moustaki A, Iliopoulou EG, Baxevanis CN Papamichail M, et al. NK cell adoptive transfer combined with Ontak -mediated regulatory $\mathrm{T}$ cell elimination induces effective adaptive antitumor immune responses. J Immunol. 2011;186:3327-35.

30. Konjevic G, Jovic V, Jurisic V, Radulovic S, Jelic S, Spuzic I. IL-2-mediated augmentation of NK-cell activity and activation antigen expression on NK- and T-cell subsets in patients with metastatic melanoma treated with interferon-alpha and DTIC. Clin Exp Metastasis. 2003;20:647-55.

31. Hershkovitz O, Rosental B, Rosenberg LA, Navarro-Sanchez ME, Jivov S, Zilka A, et al. NKp44 receptor mediates interaction of the envelope glycoproteins from the West Nile and dengue viruses with NK cells. J Immunol. 2009;183:2610-21

32. Walzel H, Blach M, Neels P, Schulz U, Wollenhaupt K, Brock J. The B-chain of mistletoe lectin I efficiently stimulates calcium signaling in human Jurkat T-cells. Immunol Lett. 2001;78:57-66. 